\title{
Randomised trial of targeted chemotherapy with lipiodol and 5-epidoxorubicin compared with symptomatic treatment for hepatoma
}

\author{
M V Madden, J E J Krige, S Bailey, S J Beningfield, C Geddes, I D Werner, J Terblanche
}

\begin{abstract}
Lipiodol injected into the hepatic artery is selectively retained in hepatomas so has been used as a vehicle for cytotoxic drugs. This study compared treatment with 5-epidoxorubicin emulsified in lipiodol and infused into the hepatic artery with symptomatic treatment alone in a randomised trial. Of 136 patients with hepatoma $78(57 \%)$ were not elegible, eight $(6 \%)$ refused to take part, and 50 entered the trial (chemotherapy: $n=25$, symptomatic treatment: $n=25$ ). The two groups had similar prognostic indices. Seven of 25 patients allocated to chemotherapy were unable to receive it. The slight survival disadvantage associated with chemotherapy was not significant (median survival 48 days compared with 51 days, $\log$ rank $\chi^{2}=0.07, p>0.05$ ). Patients given chemotherapy spent significantly longer in hospital, however (median three days compared with one, $p=0.0008$ ). Changes in symptoms and indices of tumour growth did not differ significantly between the two groups. It is concluded that infusion of 5-epidoxorubicin emulsified in lipiodol for hepatoma increased morbidity but did not affect survival. In addition, most patients were unsuitable for this treatment because of advanced disease. The patients in the trial had a short median survival time so the conclusions may not be valid for other patients with hepatoma.

(Gut 1993; 34: 1598-1600)
\end{abstract}

Hepatomas are seldom resectable and usually incurable. Iodised poppyseed oil (lipiodol) injected into the hepatic artery is selectively retained in such tumours, and there have been uncontrolled reports that an infusion of cytotoxic agents emulsified in lipiodol into the hepatic artery may increase survival of patients with hepatoma. ${ }^{1-3}$ The aim of this study was to compare the efficacy of such a regimen with our standard palliative treatment in a randomised trial.

\section{Patients and methods}

All patients seen at Groote Schuur Hospital between 1 January 1987 and 30 November 1991 who either had abnormal $\alpha$ fetoprotein estimations or who were being investigated for hepatoma were considered for inclusion in the trial. The diagnosis was confirmed if two of the following criteria were met: a 10-fold increase in serum $\alpha$ fetoprotein concentration, cytological or histological appearance compatible with a diagnosis of hepatoma; or angiographic findings that suggested hepatoma. ${ }^{4}$ Patients were eligible for the trial provided that none of the criteria listed in Table I were applicable. Spread of tumour outside the liver was sought by a chest radiograph as well as an ultrasound scan and a computed tomogram of the abdomen. Patients were randomised to receive either chemotherapy or symptomatic treatment alone by opening consecutive sealed opaque envelopes, which had been numbered according to a balanced computer generated random sequence.

The number of patients to be studied was calculated by reference to the length of survival of the first 10 cases. Two of five patients given chemotherapy and four of five who received symptomatic treatment alone survived 30 days. We wanted to be $80 \%$ certain of detecting a difference in survival of this size between the two groups and $95 \%$ sure that any difference detected was not simply a result of chance. ${ }^{5}$ A total of 46 patients was needed to establish this, and the number of cases was rounded up to 50 . The protocol remained unchanged. Survivors had computed tomograms at intervals of one month to monitor progression of the tumour.

Chemotherapy, which consisted of one dose of 5-epidoxorubicin $\left(60 \mathrm{mg} / \mathrm{m}^{2}\right)$ emulsified in $6 \mathrm{ml}$ lipiodol and $5 \mathrm{ml}$ meglumine iothalamate, was given through a catheter that had been placed in the hepatic artery during arteriography; this was repeated four weeks later if the patient still satisfied the entry criteria of the trial. The progress of the patients was evaluated monthly until they died.

The end point of the trial was length of survival after randomisation. Patients were also asked to estimate the amount of pain that they had and their appetite at entry and at each evaluation, on a linear analogue scale. The purpose, risks, and benefits associated with the trial were explained to patients who were eligible for entry by the trial coordinator using a form approved by the Ethics and Research Committee of the Medical Faculty of the University, which had also approved the trial design. The survival of the two groups was compared by the KaplanMeier log rank method. The effect of the trial's size on the validity of the findings was assessed by calculating confidence limits. ${ }^{6}$ Quantitative data that were not normally distributed were compared by Wilcoxon's rank sum test.

\section{Results}

Of the 136 patients considered for entry to the trial during the 58 months of the study, $78(57 \%)$ were not elegible; a further eight patients refused to participate (Table I). 
TABLE I Reasons for exclusion of 86 patients from the trial

\begin{tabular}{lc}
\hline & $N o(\%)$ \\
\hline ECOG stage 4 (bedridden) & $28(33)$ \\
Home too remote for follow up & $13(15)$ \\
Age $>70$ years & $11(13)$ \\
Extrahepatic tumour & $8(9)$ \\
Diagnosis not proved & $6(7)$ \\
Serious heart disease & $5(6)$ \\
Refused consent to trial & $5(6)$ \\
Chose traditional healer & $3(3)$ \\
Treated with radiotherapy & $2(2)$ \\
Tumour resected & $2(2)$ \\
Abnormal hepatic artery & $1(1)$ \\
Variceal bleeding & $1(1)$ \\
Pulmonary tuberculosis & $1(1)$ \\
\hline
\end{tabular}

Those patients who were randomised to receive chemotherapy had similar prognostic indices to those allocated to receive symptomatic treatment alone ${ }^{7}$ (Tables II, III, and IV). Patients in the chemotherapy group survived for a median of three days less than those allocated to symptomatic treatment. This difference was not significant (median 48 days (range 1-504) compared with 51 days (range $0-607$ ), log rank $\chi^{2}=$ 0.07, Kaplan-Meier, Fig). Patients who received chemotherapy spent significantly longer in hospital after diagnosis (median three days (range 1-17) compared with one (range $0-9), p=0 \cdot 0008$, Wilcoxon's rank sum test). The changes in patients' assessments of pain and appetite during their illness did not differ significantly between the two groups. The median pain score (maximum 100) in the chemotherapy group increased from 7 (range 1-99) at entry to 24 (range 6-70) after one month and from 10 (range 1-98) to 27 (range 1-89) among those who received symptomatic treatment. The median appetite score worsened from 43 (range $1-99$ ) to 16 (range 5-25) in the chemotherapy group and from 26 (range 1-99) to 19 (range 2-99) in the group that received symptomatic treatment. Objective measures of progression of the tumour did not differ between the two groups: the computed tomogram at the one month follow up showed that the tumours had increased in size in five of 25 patients in the chemotherapy group and six of 25 who had symptomatic treatment. The mean rises in serum $\alpha$ fetoprotein concentrations during the month after entry were $10 \%$ and $11 \%$, respectively.

Seven of 25 patients allocated to receive chemotherapy did not receive it (inability to cannulate the hepatic artery, $n=3$; withdrew consent for angiogram, $n=2$; coagulopathy, $\mathrm{n}=1$; early death, $\mathrm{n}=1$ ). Of the 18 patients who received an initial dose of chemotherapy three

TABLE II Comparability of the two groups

\begin{tabular}{lll}
\hline & $\begin{array}{l}\text { Chemotherapy } \\
(n=25)\end{array}$ & $\begin{array}{l}\text { Symptomatic } \\
\text { treatment } \\
(n=25)\end{array}$ \\
\hline Male:female & $21: 4$ & $25: 0$ \\
Black:mixed race:white & $14: 10: 1$ & $9: 14: 2$ \\
Median age in years (range) & $48(24-70)$ & $49(18-70)$ \\
Okuda prognostic stage: & 3 & 4 \\
$\quad$ I & 18 & 16 \\
II & 4 & 5 \\
III & $1(1-3)$ & $1(1-3)$ \\
\hline (range) & & \\
\hline
\end{tabular}

TABLE III Okuda prognostic grading *

\begin{tabular}{llll}
\hline Factor & Score & Total score & Stage \\
\hline Tumour $>50 \%$ of liver area & 1 & 0 & I \\
Ascites present & 1 & 1 or 2 & II \\
Serum albumin $<30 \mathrm{~g}$ & 1 & 3 or 4 & III \\
Serum bilirubin $>50 \mu \mathrm{m}$ & 1 & & \\
\hline
\end{tabular}

${ }^{\star}$ Modified from Okuda et al. ${ }^{7}$

remained well enough to receive a second dose one month later.

The survival of black patients $(n=23$, median 54 days, range $0-504$ ) did not differ significantly from that of the mixed race and white patients $(\mathrm{n}=27$, median 43 days, range $0-607$, log rank $\chi^{2}=0 \cdot 15$, Kaplan-Meier).

Our conclusion that the treatment is of no value is based on a small number of patients so it is important to assess the validity of the finding. The median survival time for untreated cases was 51 days while treated patients lived for a median time of 48 days. We arbitrarily assumed that a clinician would recommend the treatment if a treated patient were at least twice as likely as a control patient to survive beyond 50 days. Excluding the three patients lost to follow up, the likelihood of a treated patient living beyond 50 days was $92 \%$ of a control patient's likelihood (odds ratio 0.923 ). From the 47 patients on which this odds ratio is based, one can be $95 \%$ confident that the true odds ratio lies between 0.29 and $2 \cdot 91$. Furthermore, the likelihood of the true odds ratio being greater than 2 , when our hypothetical clinician would recommend treatment, is only 0.09 or $9 \%$. Thus despite its small size this trial shows that the likelihood that the treatment prolongs survival to a clinically important extent in hepatoma patients is fairly low.

\section{Discussion}

Targeted chemotherapy with 5-epidoxorubicin and lipiodol did not improve survival in this series of patients with hepatoma, nor did it reduce the mean tumour size or the mean serum $\alpha$ fetoprotein concentration. Assessment of the quality of life from the patients' own ratings of their pain and appetite showed that symptoms seemed to progress equally rapidly in both groups, which suggests that the chemotherapy had no placebo effect either. The significantly longer hospital stay associated with chemotherapy (three days compared with one day) may seem a trivial disadvantage, but when the median survival is only 50 days it is important. All we have shown therefore is increased morbidity associated with the chemotherapy. Previous optimistic reports of this treatment were the result of uncontrolled studies. ${ }^{1-3}$ Random control studies of embolisation with and without chemotherapy have produced little evidence that any of

TABLE IV Eastern Cooperative Oncology Group performance rating

0 Fully active

1 Ambulatory, capable of light work

In bed $<50 \%$ of the time, capable of self care but not work In bed $>50 \%$ of the time, capable of only limited self care 4 Completely bedridden 


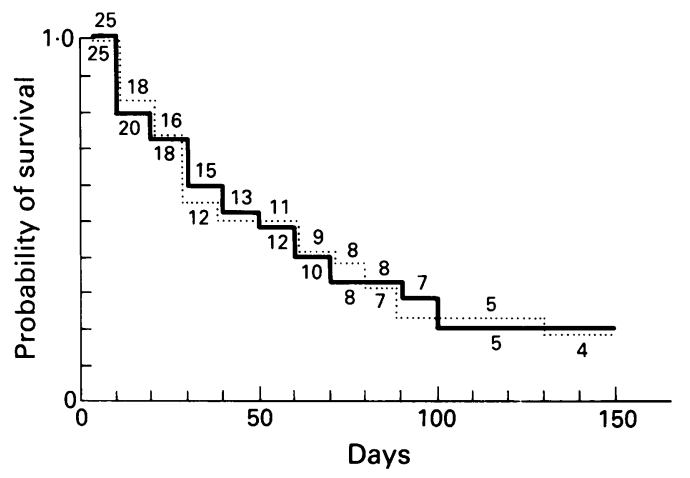

Survival of patients allocated to chemotherapy (solid line) or symptomatic treatment only (dotted line). Numbers are numbers of patients observed. Three patients in the symptomatic treatment group were lost to follow up after 1,3 and 22 days.

the treatments that have been tested are beneficial. Thus a randomised comparison in 42 patients between symptomatic treatment and embolisation with doxorubicin plus gelatin sponge showed no difference in survival. ${ }^{8} \mathrm{~A}$ random comparison between three treatment regimens in 63 patients (monthly hepatic arterial embolisation, a single embolisation followed by monthly intravenous 5-fluorouracil, and monthly intravenous 5-fluorouracil alone) showed a significantly greater likelihood of survival after treatment with repeated embolisation when compared with 5-fluorouracil alone. 9 This benefit was confined to the period from eight to 22 months after treatment and although embolisation was better than 5-fluorouracil it was not better than a single embolisation followed by 5 -fluorouracil.

Two sources of bias in this study may make it less relevant to patients with hepatoma in other countries. Firstly, $46 \%$ of the patients were black. Hepatomas in black South Africans are usually multifocal and grow rapidly. ${ }^{11}$ Those black patients who entered the trial, however, did not have a shorter survival than patients who were white or of mixed race, so their inclusion is unlikely to have biased the results. It is more important that the median survival was only $\mathbf{5 0}$ days, half of that reported for two groups of Japanese patients: about 100 days for 693 consecutive patients assessed as unsuitable for surgery $^{7}$ and about 105 days for 100 patients selected as unsuitable for any treatment. ${ }^{12}$ Moveover, it has been suggested that European patients with hepatoma may have an even better prognosis. ${ }^{813}$ The short survival of patients in this study may mean that our conclusions do not apply to other patients with hepatomas.

In this study chemotherapy with 5-epidoxorubicin emulsified in lipiodol did not improve survival and increased hospital stay in patients with hepatoma. Although this trial is small, the likelihood that it failed to detect a clinically useful improvement in survival caused by treatment is quite low. The patients studied, however, had a short survival time so this conclusion may not be valid for all patients with this tumour. The treatment was not applicable to half of the patients, usually because the tumour was too advanced at the time of diagnosis.

We are grateful to Dr Deborah Bradshaw (Centre for Epidemiological Research in Southern Africa, Medical Research Council of South Africa) for calculating the number of patients to be studied and for assessing the effect of the trial's size on the validity of the results.

These data were presented in part at the July 1992 meeting of the South African Gastroenterology Society.

1 Kanematsu T, Inokuchi K, Sugimachi K, Furuta T, Sonoda $\mathrm{T}$, Tamura S, et al. Selective effects of lipiodolised antitumo agents. F Surg Oncol 1984; 25: 218-26.

2 Kanematsu T, Furuta T, Takenaka K, Matsumata T, Yoshida Y, Nishizaki T, et al. A 5-year experience of lipiodolisation: selective regional chemotherapy for 200 patients with hepatocellular carcinoma. Hepatology 1989; 10: 98-102.

3 Kirk S, Blumgart R, Craig B, Rosen A, Terblanche J, Spence RAJ. Irresectable hepatoma treated by intrahepatic iodized oil doxorubicin hydrochloride: Initial results. Surgery 1991; 109: 694-7.

4 Okuda K. Diagnosis and non-surgical treatment of hepatocellular carcinoma. Hepatogastroenterology 1990; 37: 159-64. Gail M, Gart JJ. The determination of sample sizes for use with the exact conditional test in $2 \times 2$ comparative trials Biometrics 1973; 29: 441-8.

6 Smith AH, Bates MN. Confidence limit analyses should replace power calculations in the interpretation of epidemiologic studies. Epidemiology 1992; 3: 449-52.

7 Okuda K, Ohtsuki T, Obata H, Tommimatsu M, Okazaki N, Hasegawa $\mathrm{H}$, et al. Natural history of hepatocellular carcinoma and prognosis in relation to treatment. Study of 850 patients. Cancer $1985 ; 56$ : $918-28$.

8 Pelletier G, Roche A, Ink O, Anciaux ML, Derhy S, Rougie $P$, et al. A randomised trial of hepatic arterial chemoembolisation in patients with unresectable hepatocellular embolisation in patients with unresecta

9 Lin D-Y, Liaw Y-F, Lee T-Y, Lai C-M. Hepatic arterial embolisation in patients with unresectable hepatocellular carcinoma - a randomised controlled trial. Gastroenterolog 1988; 94: 453-6.

10 Wands JR, Blum HE. Primary hepatocellular carcinoma NEnglf Med 1991; 325: 729-31.

11 Anonymous. Hepatocellular cancer: differences between high and low incidence regions [Editorial]. Lancet 1987; ii: 1183-4

12 Nagasue N, Yukaya H, Hamada T, Hirose S, Kanashima R, Inokuchi $\mathrm{K}$. The natural history of hepatocellular carcinoma. A study of 100 untreated cases. Cancer 1984; 54: 1461-5.

13 Forbes A, Williams R. Chemotherapy and radiotherapy of malignant hepatic tumours. Balliéres Clin Gastroenterol 1987; 1: 151-69. 